\title{
GENERATION AND IMPLICATIONS OF POST-MERGER TURBULENCE IN CLUSTERS OF GALAXIES
}

\section{GOLDMAN}

School of Physics and Astronomy, Tel Aviv University, Tel Aviv 69978, Israel; goldman@post.tau.ac.il

\section{Introduction}

Observations in X-ray and optical suggest that mergers of sub-clusters with galaxy clusters are quite common (for Coma see e.g., White et al. 1993; Colless \& Dunn 1996; Ishizaka \& Mineshige 1996). A merger leads to violent relaxation of the dissipationless dark matter resulting in a time-dependent gravitational potential. This in turn generates large-scale flows and shocks in the collisional baryonic intracluster gas (Takizawa \& Mineshige 1997). Both the large scale flows and the shocks will excite turbulence in the gas. We focus here on turbulence generated by shocks, which is less dependent on the specifics of the merger. This paper is based on a more detailed work (Goldman 1997).

\section{Intracluster Turbulence Generated by Shocks}

For a shocked region of size $\sim 0.8 \mathrm{Mpc}$ and merging velocities of $\sim 1000 \mathrm{~km}$ $\mathrm{s}^{-1}$, we obtain for the largest turbulence scale and the corresponding turbulent velocity: $l_{0} \sim 250 \mathrm{Kpc}$ and $v_{0}=v_{t u r b}\left(l_{0}\right) \sim 250 \mathrm{~km} \mathrm{~s}^{-1}$, respectively. The turbulence timescale is thus $\tau_{0} \sim \frac{l_{0}}{v_{0}} \sim 1 \mathrm{Gyr}$.

The energy input to the turbulence is mainly to the largest eddies. The non-linear eddy interactions result in an energy cascade to the small scales, down to a scale where it is dissipated. Over more than a decade in wavenumbers, the spectrum is a power-law $v(l)=v_{0}\left(\frac{l}{l_{0}}\right)^{m}$ with the Kolmogorov value $m=1 / 3$. In case of stable stratification the spectrum is quasi two dimensional with $m=1$. In case that the local rotation rate is high, $m=1 / 2$. 
The heating rate per unit mass is $\epsilon \sim \frac{v_{0}^{3}}{l_{0}} \sim 0.02 \mathrm{erg} \mathrm{gr}^{-1} \mathrm{~s}^{-1}$ and the resulting luminosity is $L_{t u r b}=\epsilon M_{m g} \sim 4 \times 10^{44}\left(\frac{M_{m g}}{10^{13} M_{\odot}}\right) \mathrm{erg} \mathrm{s}^{-1}$ with $M_{m g}$ denoting the merged gas mass. Thus, the turbulence is potentially an important heat source for the intracluster gas.

The turbulence timescale, on spatial scale $l$, is $\tau(l) \sim \frac{l}{v(l)}$. For $l=10$ $\mathrm{Kpc}$ and Kolmogorov spectrum it is $\sim 10^{8}$ yrs. Given that the available time for the turbulence is $\tau_{0} \sim 10^{9} \mathrm{yrs}$, amplification of magnetic fields by a large factor is possible. If the magnetic field is amplified to equipartition with $v(l)$, it can reach a level of $\sim 1 \mu \mathrm{G}$.

Turbulent heat conduction and turbulent mixing will be effective in smoothing out temperature and abundance gradients over a spatial scale $l<l_{0}$, if the available time exceeds $\tau(l)$. For truly stationary turbulence this could have occurred also on scales $>l_{0}$, provided time spans exceeding $\tau_{0}$ were available. However, the lifetime of the turbulence is itself of the order of $\tau_{0}$. Thus, one may expect that scales $\leqslant l_{0} \sim 250 \mathrm{Kpc}$ will be affected.

\section{Concluding Remarks}

The observations suggest occurrence of multiple mergers, so a turbulent region could be shocked again. As a result, the largest turbulence scale will decrease by $\sim 1.4$ and the corresponding turbulent velocity will increase by a similar factor. This will increase the heating rate of the intracluster gas by a factor of $\sim 4$, will shorten the turbulent timescales by a factor of $\sim 2$ and thus could boost up magnetic field amplification.

Future X-ray spectrometers with high spectral and spatial resolutions could measure the underlying turbulence spectrum and thus decide on its nature. This could help to constrain the parameters of the merger, notably the amount of angular momentum in the merged gas, and stratification.

If a strong enough magnetic field is present, it can trap the cascaded turbulent energy before it reaches the viscous scales, and thus generate a magnetic turbulence. In such a case, a change in the turbulence spectrum is expected to be observed at a scale $\gtrsim 10 \mathrm{Kpc}$.

This work was supported by the US-Israel BSF grant 94-314.

\section{References}

Colless, M. \& Dunn, A.M. 1996, ApJ, 458, 435

Goldman, I. 1997, in preparation

Ishizaka, C. \& Mineshige, S. 1996, PASJ, 48, L37

Takizawa, M. \& Mineshige, S. 1997, astro-ph/9702047

White, S.D.M., Briel, U.G. \& Henry, J.P. 1993, MNRAS, 261, L8 